\title{
Quantification of optical absorption coefficient from acoustic spectra in the optical diffusive regime using photoacoustic microscopy
}

Zijian Guo, Christopher Favazza, Lihong V. Wang

Zijian Guo, Christopher Favazza, Lihong V. Wang, "Quantification of optical absorption coefficient from acoustic spectra in the optical diffusive regime using photoacoustic microscopy," Proc. SPIE 8223, Photons Plus Ultrasound: Imaging and Sensing 2012, 82234C (23 February 2012); doi: $10.1117 / 12.906787$

SPIE. Event: SPIE BiOS, 2012, San Francisco, California, United States 


\title{
Quantification of optical absorption coefficient from acoustic spectra in the optical diffusive regime using photoacoustic microscopy
}

\author{
Zijian Guo, Christopher Favazza, and Lihong V. Wang* \\ Optical Imaging Laboratory, Department of Biomedical Engineering, Washington University \\ 1 Brookings Drive, Saint Louis, Missouri 63130, USA \\ *Corresponding author: 1hwang@biomed.wustl.edu
}

\begin{abstract}
Photoacoustic (PA) tomography (PAT) can image optical absorption contrast with ultrasonic spatial resolution in the optical diffusive regime. Multi-wavelength PAT can noninvasively monitor hemoglobin oxygen saturation $\left(\mathrm{sO}_{2}\right)$ with high sensitivity and fine spatial resolution. However, accurate quantification in PAT requires knowledge of the optical fluence distribution, acoustic wave attenuation, and detection system bandwidth. We propose a method to circumvent this requirement using acoustic spectra of PA signals acquired at two optical wavelengths. With the acoustic spectral method, the absorption coefficients of an oxygenated bovine blood phantom at 560 and $575 \mathrm{~nm}$ were quantified with errors of $<5 \%$.
\end{abstract}

Keywords: Photoacoustic tomography, acoustic spectrum, hemoglobin oxygen saturation

\section{INTRODUCTION}

Oxygenated and deoxygenated hemoglobin concentrations $\left(\left[\mathrm{HbO}_{2}\right]\right.$ and $\left.[\mathrm{HbR}]\right)$ as well as the derived quantitiestotal hemoglobin concentration ([HbT]) and hemoglobin oxygen saturation $\left(\mathrm{sO}_{2}=\left[\mathrm{HbO}_{2}\right] /[\mathrm{HbT}]\right)$ - are important physiological parameters. Several techniques have been developed to quantify hemoglobin concentrations in vivo; however, they all have disadvantages. Diffuse optical tomography (DOT) has poor spatial resolution due to strong scattering in biological tissue; blood oxygen level dependent (BOLD) contrast magnetic resonance imaging (MRI) can monitor only $[\mathrm{HbR}]$. Positron emission tomography (PET) measures only oxygen metabolism, and they not only suffer from poor spatial resolution but also require intravenous administration of radioactive isotopes (1).

Photoacoustic (PA) tomography (PAT) can quantify hemoglobin concentrations in vivo based on endogenous contrast with both fine spatial resolution and high sensitivity (2). In PAT, the sample is typically illuminated by a pulsed laser. Following the absorption of optical energy, an initial pressure is generated via thermo-elastic expansion. The initial pressure then propagates as ultrasonic waves, which are detected by ultrasonic sensors. The strength of the initial pressure $P_{0}(\vec{r})$ in the unit of $\mathrm{Pa}$ at the location $\vec{r}$ in the biological tissue is proportional to the local absorbed optical energy density $A(\vec{r})$ in units of $\mathrm{J} \mathrm{m}^{-3}$. From multi-wavelength PA measurements, we can obtain the optical spectrum $A(\vec{r}, \lambda)$ [i.e., $A(\vec{r})$ versus optical wavelength $\lambda$ ], which can be used to quantify hemoglobin concentrations in the same way as DOT (3). In the optical ballistic regime, the lateral resolution of PAT is determined by optical focusing (4), and thus it is comparable to that of other optical microscopy modalities. In the optical diffusive regime, however, the resolution of PAT is determined by ultrasonic waves (5), and PAT provides much better spatial resolution than DOT, in which the inverse algorithm is ill-posed. While DOT can only monitor $\mathrm{sO}_{2}$ which is volume-averaged over multiple blood vessels, PAT can pinpoint blood vessels and evaluate their individual $\mathrm{sO}_{2}$ levels (6). Moreover, PAT inherently exploits optical absorption contrast, and thus it has a much higher sensitivity to optical absorption than other optical microscopy modalities (7) and DOT.

Photons Plus Ultrasound: Imaging and Sensing 2012, edited by Alexander A. Oraevsky, Lihong V. Wang, Proc. of SPIE Vol. 8223, 82234C · (C) 2012 SPIE · CCC code: 1605-7422/12/\$18 - doi: 10.1117/12.906787 
Nevertheless, using PAT to quantify hemoglobin concentrations conventionally requires knowledge of the local optical fluence $(8,9)$. In the quantification model, hemoglobin concentrations are derived (3) from the optical absorption coefficient $\mu_{a}(\vec{r}, \lambda)$ in the unit of $\mathrm{m}^{-1}$ by solving the following equation for multiple values of $\lambda$ :

$$
\mu_{a}(\vec{r}, \lambda)=\varepsilon_{\mathrm{HbR}}(\lambda)[\mathrm{HbR}](\vec{r})+\varepsilon_{\mathrm{HbO}_{2}}(\lambda)\left[\mathrm{HbO}_{2}\right](\vec{r}),
$$

where $\varepsilon_{\mathrm{HbR}}(\lambda)$ and $\varepsilon_{\mathrm{HbO}_{2}}(\lambda)$ are the known molar extinction coefficients $\left(\mathrm{m}^{-1} \mathrm{M}^{-1}\right)$ of deoxyhemoglobin $(\mathrm{HbR})$ and oxyhemoglobin $\left(\mathrm{HbO}_{2}\right)$ at wavelength $\lambda$. Apart from spatial variation of the Grueneisen coefficient $(\Gamma)(2)$, PAT images, however, are spatial mappings of the absorbed optical energy density $A(\vec{r}, \lambda)=\mu_{a}(\vec{r}, \lambda) F(\vec{r}, \lambda)$, where $F(\vec{r}, \lambda)$ represents the local optical fluence in units of $\mathrm{J} \mathrm{m}^{-2}$. To obtain the intrinsic quantity $\mu_{a}(\vec{r}, \lambda)$ from $A(\vec{r}, \lambda)$, we need to quantify $F(\vec{r}, \lambda)$, which is usually unknown due to light transport in the scattering tissue.

$F(\vec{r}, \lambda)$ can be quantified in vivo either invasively (1) by inserting a calibration target or non-invasively (10-13) by using an auxiliary modality. In the invasive method, an exogenous optical absorber with known absorption spectrum $\mu_{a}\left(\vec{r}^{\prime}, \lambda\right)$ is inserted at $\vec{r}^{\prime}$ near the target objects of interest. The PA amplitudes due to $A(\vec{r}, \lambda)$ of the target objects of interest are normalized by the PA amplitude due to $A\left(\vec{r}^{\prime}, \lambda\right)$ of the calibration target. By assuming $F\left(\vec{r}^{\prime}, \lambda\right) \approx F(\vec{r}, \lambda)$, we have $\mu_{a}(\vec{r}, \lambda)=\mu_{a}\left(\vec{r}^{\prime}, \lambda\right) A(\vec{r}, \lambda) / A\left(\vec{r}^{\prime}, \lambda\right)$. This method physically compensates for the fluence attenuation; however, it is invasive. The non-invasive method involves solving both the optical diffusion equation and the PA wave equation iteratively. However, additional optical measurements are required, and the inverse algorithms are ill-posed.

The temporal profile of the PA signal has also been used to quantify optical absorption coefficients with reflectionmode PA imaging systems (14-17). For example, if a pencil beam incident perpendicularly on the blood vessel surface, then the energy deposition in the vessel decays exponentially along the beam propagation direction. If at wavelength $\lambda$ the reduced scattering coefficient $\mu_{s}{ }^{\prime}(\lambda)$ is much smaller than the absorption coefficient $\mu_{a}(\lambda)$ of blood, fitting the received PA signals with Beer's law yields $\mu_{a}(\lambda)$. Here, knowledge of the local optical fluence is not required, because $\mu_{a}(\lambda)$ is quantified from the relative temporal decay profile. However, various acoustic effects may distort the received PA signals. Acoustic attenuation in biological tissue has a power law dependence on the frequency, and therefore the shapes of the acoustic pulses change as they propagate. Also, as ultrasonic detectors have limited bandwidths, the detected PA signal is the convolution of the received acoustic pulse and the mechanical-electrical impulse response of the detector. As such, the temporal profiles no longer follow the exponential decay. Consequently, directly fitting the temporal profile for optical absorption coefficients can be inaccurate.

We propose a method to quantify optical absorption coefficients from the acoustic spectra of PA signals at multiple optical wavelengths for two main reasons. First, similar to fitting the PA temporal profiles for $\mu_{a}(\lambda)$, quantification of $\mu_{a}(\lambda)$ from acoustic spectra does not require the knowledge of the local optical fluence. Second, the response function of the ultrasonic detection system and the acoustic properties of the tissue are independent of the optical wavelength; therefore, their effects can be eliminated mathematically in the inverse problem of quantifying optical properties.

\section{METHODS}

Mathematically, the acoustic spectrum $S(\omega, \lambda)$ of the received PA signal from an absorbing target object of interest can be written, based on the assumptions of linearity and shift-invariance, as (18)

$$
S(\omega, \lambda)=F_{I}(\lambda) O(\omega, \lambda) a(\omega) H(\omega)
$$


Here, $\lambda$ is the optical wavelength. If the acoustic spectra are measured at multiple optical wavelengths, we have $\lambda=\lambda_{i}$ ( $i=1,2, \cdots n$ ), where $n$ is the total number of optical wavelengths. $\omega$ is the acoustic angular frequency, $F_{I}(\lambda)$ is the optical fluence incident on the target object, $a(\omega)$ is the acoustic attenuation in the tissue between the target object and the ultrasonic transducer, and $H(\omega)$ is the system transfer function of the ultrasonic detection system. Since $a(\omega)$ and $H(\omega)$ are independent of the optical wavelength $\lambda$, and $F_{I}(\lambda)$ is independent of the acoustic frequency $\omega$, these factors can be eliminated, as will be shown later. $O(\omega, \lambda)$ represents the original acoustic spectrum of the target object of interest (e.g., a blood vessel), and it equals to the Fourier transform of $O(t, \lambda)$, the time-domain PA signal from the target object of interest measured locally with an ideal ultrasonic detection system of infinite bandwidth at unit optical fluence. Here, $t$ denotes time. $O(t, \lambda)$ is related to the target object's shape, size, optical properties, and the incident fluence distribution. If $O(\omega, \lambda)$ is recovered, $O(t, \lambda)$ becomes known, and subsequently $\mu_{a}(\lambda)$ can be quantified through an appropriate model of $O(t, \lambda)$.

We applied this idea for the acoustic-resolution PA microscopy (AR-PAM) (6). Figure 1(a) shows the AR-PAM system. A dye laser pumped by a Nd:YLF laser served as the irradiation source. At each location, a focused ultrasonic transducer was employed to record the PA wave, which was converted into a one-dimensional (1D) depth-resolved image (A-scan or A-line). A three-dimensional (3D) image was achieved by raster scanning in the $\mathrm{x}-\mathrm{y}$ plane. The lateral resolution of the AR-PAM, determined by the focal diameter of the ultrasonic transducer, was $\sim 45 \mu \mathrm{m}$ for the $50 \mathrm{MHz}$ transducer.

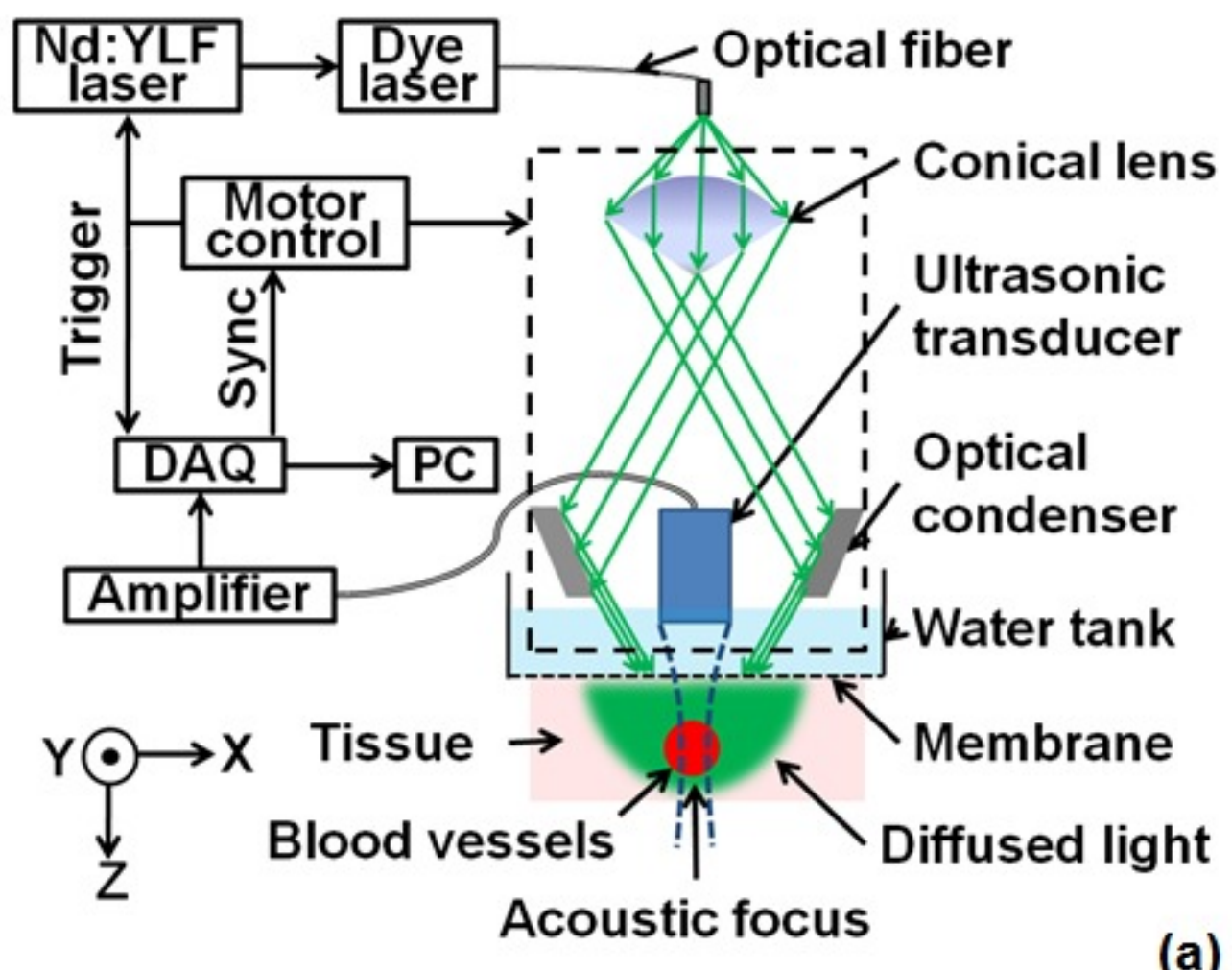




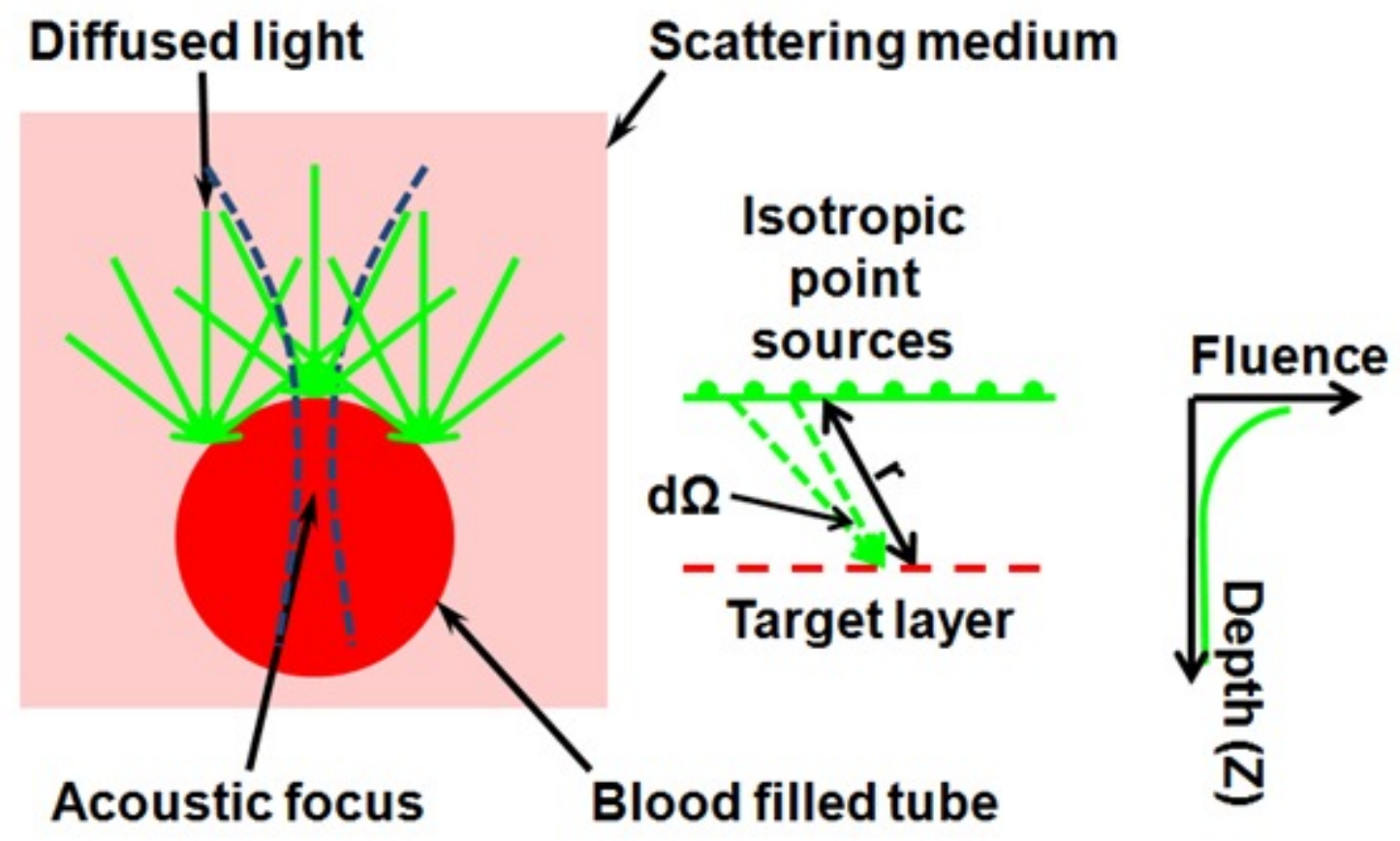

(b)

Fig. 1. Schematic of the AR-PAM system and phantom experiment setups. (a) The schematic of the AR-PAM system. A dye laser pumped by a Nd:YLF laser was the irradiation source. The laser beam from the dye laser was delivered through an optical fiber and passes through a conical lens to provide a ring-shaped area of illumination. A focused ultrasonic transducer was employed to detect PA waves. (b) Use AR-PAM to image a blood-filled tube inserted $1.5 \mathrm{~mm}$ deep in the optical scattering medium. The fluence decay profile in the blood is modeled.

With AR-PAM, however, several assumptions can simplify the modeling of $O(t, \lambda)$. First, the surface of blood vessels with sufficiently large diameters (e.g., greater than $300 \mu \mathrm{m}$ for the $45 \mu \mathrm{m}$ lateral resolution) may be treated approximately as a flat surface locally. Second, when imaging blood vessels at depths greater than one transport mean free path $\left(l_{t}{ }^{\prime} \sim 1 \mathrm{~mm}\right.$ in biological tissue), we can assume that the light is completely diffused and can be considered as isotropic point sources [Fig. 1(b)]. The fluence in the blood vessel can be expressed as

$$
F(z, \lambda)=F_{I}(\lambda) \int_{\Omega} \exp \left[-\mu_{a}(\lambda) r\right] \frac{d \Omega}{2 \pi},(3)
$$

where $z$ is the depth of the target layer in the blood vessel, $r$ is the distance between the isotropic point source and one point in the layer at the depth of $z$, and $F_{I}(\lambda)$ is the incident fluence on the blood vessel. $d \Omega=\sin \theta d \theta d \varphi$ is the differential solid angle in the spherical coordinates, and the integration ranges of the polar angle $\theta$ and the azimuthal angle $\varphi$ are $[0, \pi / 2]$ and $[0,2 \pi]$, respectively. In blood, $\mu_{s}{ }^{\prime}(\lambda)$ is much less than $\mu_{a}(\lambda)$ [e.g., $1.5 \mathrm{~cm}^{-1}<<241.0$ $\mathrm{cm}^{-1}$ at $\left.571 \mathrm{~nm}(19)\right]$, because the anisotropy factor $(g)$ is so close to 1 in the optical spectral region we used (around $585 \mathrm{~nm})(19)$; therefore scattering is negligible. Equation (3) can be further simplified to

$$
F(z, \lambda)=F_{I}(\lambda) \int_{0}^{1} \exp \left[-\mu_{a}(\lambda) z / u\right] d u,
$$


where $u$ is a dimensionless scaling factor defined by $u=z / r$. Equation (4) was validated by Monte Carlo simulations (20) (see Appendix B). The original PA signal from the target object induced by unit incident optical fluence is expressed as

$$
O(t, \lambda)=\Gamma \mu_{a}(\lambda) F(z, \lambda) / F_{I}(\lambda)=\Gamma \mu_{a}(\lambda) \int_{0}^{1} \exp \left[-\mu_{a}(\lambda) c t / u\right] d u,(5)
$$

where $z$ is converted to time $t$ through the speed of sound $c: t=z / c$.

An algorithm, named the absorption-fitting algorithm, was developed to fit for the optical absorption coefficients as well as the relative incident fluence from the acoustic spectra at multiple wavelengths. We normalize the acoustic spectrum $S\left(\omega, \lambda_{i}\right) \quad(i=1,2, \cdots n)$ at each optical wavelength by the root-mean-square spectrum

$$
S_{\mathrm{rms}}(\omega)=\sqrt{\sum_{i=1}^{n}\left|S\left(\omega, \lambda_{i}\right)\right|^{2} / n} \text { as }
$$

$$
\bar{S}\left(\omega, \lambda_{i}\right)=\frac{S\left(\omega, \lambda_{i}\right)}{S_{\mathrm{rms}}(\omega)}=\frac{F_{I}\left(\lambda_{i}\right) O\left(\omega, \lambda_{i}\right)}{\sqrt{\sum_{i=1}^{n}\left|F_{I}\left(\lambda_{i}\right) O\left(\omega, \lambda_{i}\right)\right|^{2} / n}} . \text { (6) }
$$

The effects of both the system-dependent response $H(\omega)$ and the tissue related acoustic attenuation $a(\omega)$ are cancelled in the normalization process. The optical absorption coefficient and the relative incident fluence at each optical wavelengths $\lambda_{i}(i=1,2, \cdots n)$ are optimized by minimizing the following cost function:

$$
\sum_{i=1}^{n} \int\left|\bar{S}_{\exp }\left(\omega, \lambda_{i}\right)-\bar{S}_{\text {model }}\left(\omega, \lambda_{i}\right)\right|^{2} d \omega . \text { (7) }
$$

Here, $\bar{S}_{\exp }\left(\omega, \lambda_{i}\right)$ is calculated from the experimentally measured acoustic spectra using Eq. $(6) ; \bar{S}_{\text {model }}\left(\omega, \lambda_{i}\right)$ is computed from our theoretical model containing the fitting parameters $\mu_{a}\left(\lambda_{i}\right)$ and $F_{I}\left(\lambda_{i}\right)$ using Eq. (5), Fourier transformation, and Eq. (6). The minimization process is accomplished with a non-linear optimization algorithm-the Levenberg-Marquardt algorithm (LMA) - available as a standard optimization package in Matlab.

In special circumstances, when the compositions of the absorbing medium are known, we can directly fit the acoustic spectra for the concentrations of the optical absorbers. For example, in blood the major optical absorbers are $\mathrm{HbO}_{2}, \mathrm{HbR}$, and water. In the optical spectral region we used (around $585 \mathrm{~nm}$ ), the absorption of water is negligible in comparison to that of $\mathrm{HbO}_{2}$ and $\mathrm{HbR}$. An algorithm, named the concentration-fitting, was developed to directly fit for the hemoglobin concentrations $\left(\left[\mathrm{HbO}_{2}\right]\right.$ and $\left.[\mathrm{HbR}]\right)$ in blood. In the cost function [Eq. (7)], we substitute the optical absorption coefficient at $\lambda_{i}$ by $\mu_{a}\left(\lambda_{i}\right)=\varepsilon_{\mathrm{HbO}_{2}}\left(\lambda_{i}\right)\left[\mathrm{HbO}_{2}\right]+\varepsilon_{\mathrm{HbR}}\left(\lambda_{i}\right)[\mathrm{HbR}]$. Then $\left[\mathrm{HbO}_{2}\right],[\mathrm{HbR}]$, and $F_{I}\left(\lambda_{i}\right)$ are optimized from Eq. (7) by the LMA.

In a phantom study, we used AR-PAM to image a fully oxygenated bovine blood phantom with two wavelengths (560 and $575 \mathrm{~nm}$ ). The blood phantom was in a $1 \mathrm{~mm}$ diameter tube, which was located $1.5 \mathrm{~mm}$ deep in the optical scattering medium (10\% gelatin, $1 \%$ intralipid, $\left.5 \% \mathrm{CuCl}_{2}\right)$ and parallel to its surface [Fig. 1(b)].

\section{RESULTS}

In the phantom study, PA A-line signals at two wavelengths were acquired from the samples [Fig. 2(a)]. The corresponding acoustic spectra of the PA signals $[S(\omega, \lambda)]$ were calculated [Fig. 2(b)]. Light penetrated deeper at longer wavelengths, and the corresponding PA signals decayed more slowly in the time domain. At $575 \mathrm{~nm}$, the fluence decayed faster in the tube, and the produced PA signals were sharper with time. Therefore, the acoustic spectra acquired at longer optical wavelengths contained more high-frequency components. 
By fitting the acoustic spectra with the absorption-fitting algorithm, we found the absorption coefficients at two wavelengths [Fig. 2(c)]. In comparison to the gold standard-spectrophotometry, the absorption-fitting algorithm quantified the absorption coefficients with relative errors of $1.7 \%$ and $1.9 \%$ at the optical wavelengths of 560 and 575 nm, respectively [Fig. 2(c)], where the error bars indicate the fitting standard errors.

(a)

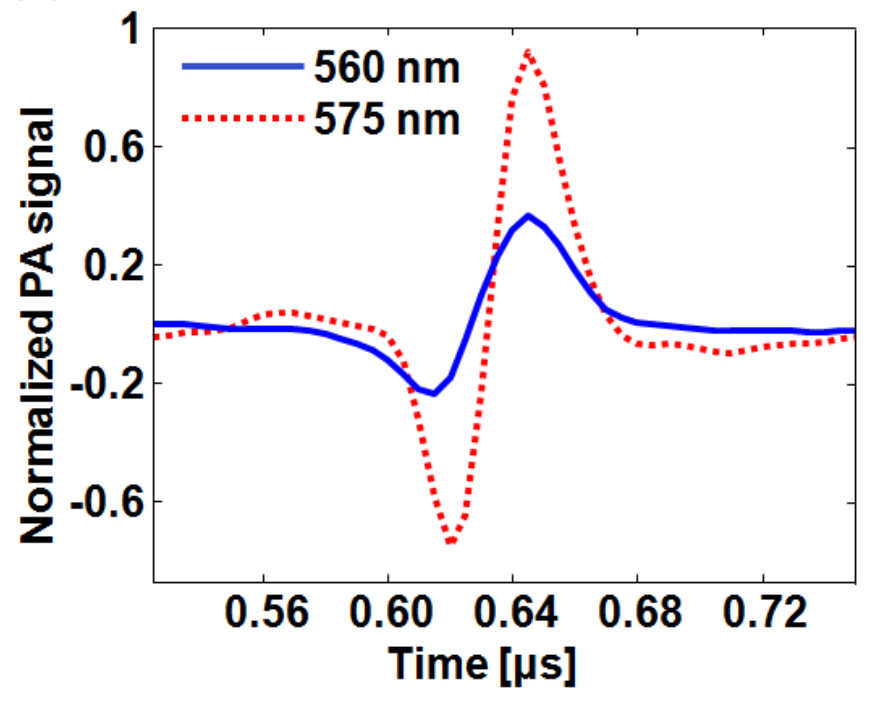

(b)

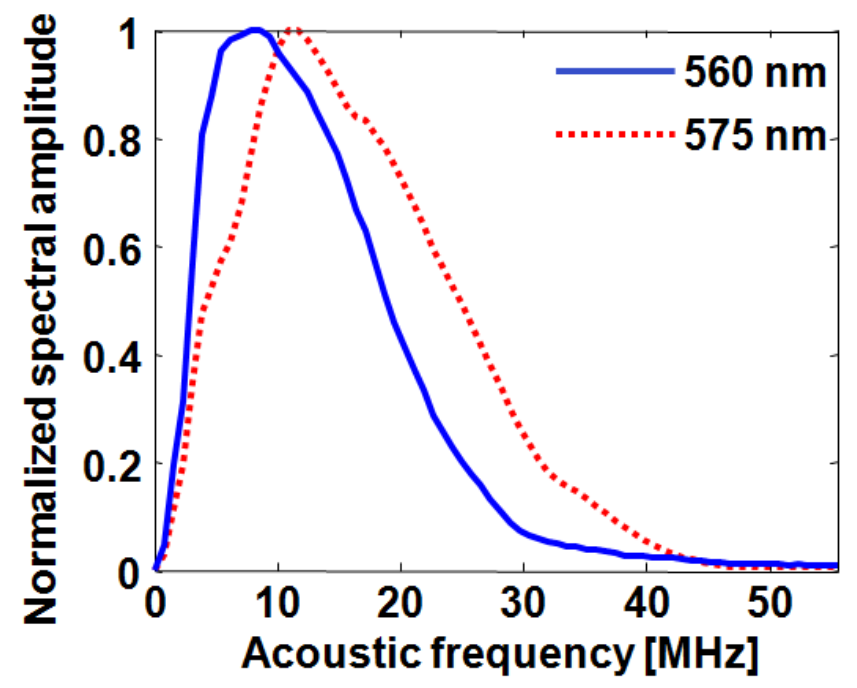


(c)

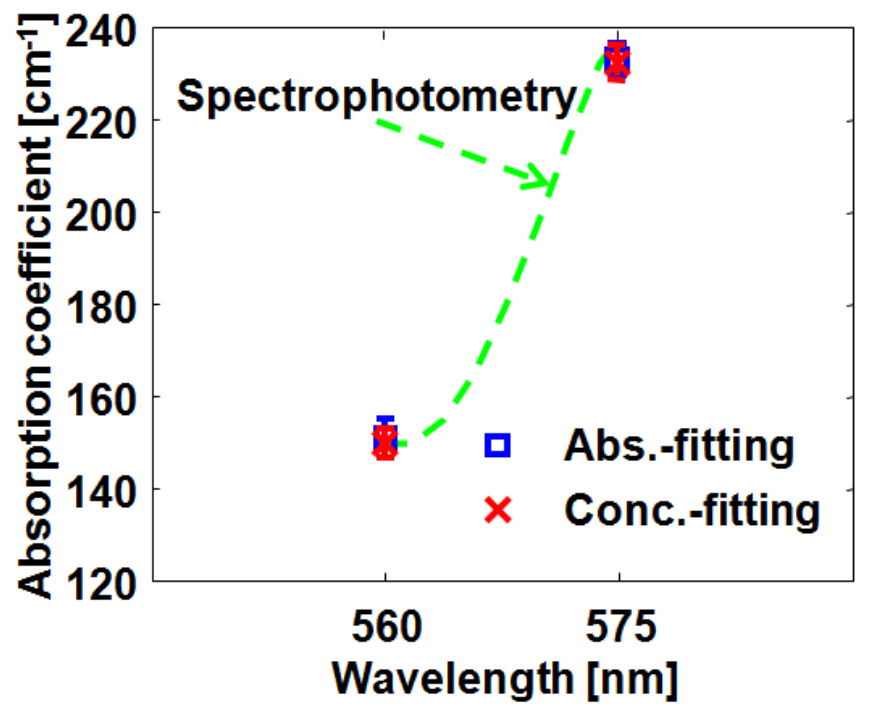

Fig. 2. Quantification of the optical absorption coefficients and hemoglobin concentrations of oxygenated bovine blood phantom. (a) PA time domain A-scan signals of the oxygenated-bovine-blood-filled tube phantom buried in the scattering medium. (b) Acoustic spectra of the PA A-scan signals. (c) Comparison of the optical absorption coefficients quantified with the acoustic spectral method and measured by spectrophotometry.

\section{CONCLUTION}

In summary, we have proposed a method to quantify $[\mathrm{HbT}]$ and $\mathrm{sO}_{2}$ in vivo using acoustic spectra of PA signals from multiple optical wavelength measurements. The optical and acoustic effects that affect the quantification accuracy have been eliminated. Using AR-PAM, we demonstrated this method with phantom experiments. The acoustic spectral method provides greater quantification accuracy than the conventional amplitude method in the optical diffusive regime. This method can potentially be applied to all other reflection- or transmission-mode PAT setups.

\section{ACKNOWLEDGMENTS}

We would like to thank Yan Liu, Dr. Xiao Xu and Dr. Konstantin Maslov for useful discussions and Prof. James Ballard for editing the manuscript. This work was sponsored in part by National Institutes of Health grants R01 EB008085, R01 EB000712, R01 CA134539, R01 CA157277, and U54 CA 136398. L. W. has a financial interest in Microphotoacoustics, Inc. and Endra, Inc., which, however, did not support this work. 


\section{REFERENCES}

1. H. F. Zhang, K. Maslov, M. Sivaramakrishnan, G. Stoica and L. H. V. Wang, "Imaging of hemoglobin oxygen saturation variations in single vessels in vivo using photoacoustic microscopy," Applied Physics Letters 90(5), 053901 (2007)

2. $\quad$ L. V. Wang and H. Wu, Biomedical Optics: Principles and Imaging, Wiley, Hoboken, NJ (2007).

3. B. Chance, E. Borer, A. Evans, G. Holtom, J. Kent, M. Maris, K. Mccully, J. Northrop and M. Shinkwin, "Optical and Nuclear-Magnetic-Resonance Studies of Hypoxia in Human Tissue and Tumors," Annals of the New York Academy of Sciences 551(1-16 (1988)

4. K. Maslov, H. F. Zhang, S. Hu and L. V. Wang, "Optical-resolution photoacoustic microscopy for in vivo imaging of single capillaries," Opt Lett 33(9), 929-931 (2008)

5. $\quad$ L. V. Wang, "Multiscale photoacoustic microscopy and computed tomography," Nature photonics 3(9), 503509 (2009)

6. H. F. Zhang, K. Maslov, G. Stoica and L. H. V. Wang, "Functional photoacoustic microscopy for highresolution and noninvasive in vivo imaging," Nature Biotechnology 24(7), 848-851 (2006)

7. L. V. Wang, "Tutorial on photoacoustic microscopy and computed tomography," Ieee Journal of Selected Topics in Quantum Electronics 14(1), 171-179 (2008)

8. K. Maslov, H. F. Zhang and L. V. Wang, "Effects of wavelength-dependent fluence attenuation on the noninvasive photoacoustic imaging of hemoglobin oxygen saturation in subcutaneous vasculature in vivo," Inverse Problems 23(6), S113-S122 (2007)

9. B. T. Cox, J. G. Laufer and P. C. Beard, "The challenges for quantitative photoacoustic imaging," in Proc. SPIE (2009).

10. J. Laufer, D. Delpy, C. Elwell and P. Beard, "Quantitative spatially resolved measurement of tissue chromophore concentrations using photoacoustic spectroscopy: application to the measurement of blood oxygenation and haemoglobin concentration," Physics in Medicine and Biology 52(1), 141-168 (2007)

11. Z. Yuan and H. B. Jiang, "Quantitative photoacoustic tomography: Recovery of optical absorption coefficient maps of heterogeneous media," Applied Physics Letters 88(23), 231101 (2006)

12. A. Q. Bauer, R. E. Nothdurft, T. N. Erpelding, L. V. Wang and J. P. Culver, "Quantitative photoacoustic imaging: correcting for heterogeneous light fluence distributions using diffuse optical tomography," J Biomed Opt 16(9), 096016 (2011)

13. P. D. Kumavor, C. Xu, A. Aguirre, J. Gamelin, Y. Ardeshirpour, B. Tavakoli, S. Zanganeh, U. Alqasemi, Y. Yang and Q. Zhu, "Target detection and quantification using a hybrid hand-held diffuse optical tomography and photoacoustic tomography system," J Biomed Opt 16(4), 046010 (2011)

14. R. O. Esenaliev, I. V. Larina, K. V. Larin, D. J. Deyo, M. Motamedi and D. S. Prough, "Optoacoustic technique for noninvasive monitoring of blood oxygenation: a feasibility study," Applied Optics 41(22), 4722-4731 (2002)

15. J. Laufer, C. Elwell, D. Delpy and P. Beard, "In vitro measurements of absolute blood oxygen saturation using pulsed near-infrared photoacoustic spectroscopy: accuracy and resolution," Physics in Medicine and Biology 50(18), 4409-4428 (2005)

16. Y. Y. Petrov, I. Y. Petrova, I. A. Patrikeev, R. O. Esenaliev and D. S. Prough, "Multiwavelength optoacoustic system for noninvasive monitoring of cerebral venous oxygenation: a pilot clinical test in the internal jugular vein," Optics Letters 31(12), 1827-1829 (2006)

17. Y. Wang and R. K. Wang, "Photoacoustic recovery of an absolute optical absorption coefficient with an exact solution of a wave equation," Physics in Medicine and Biology 53(21), 6167-6177 (2008)

18. Z. Guo, S. Hu and L. V. Wang, "Calibration-free absolute quantification of optical absorption coefficients using acoustic spectra in 3D photoacoustic microscopy of biological tissue," Opt Lett 35(12), 2067-2069 (2010)

19. A. Roggan, M. Friebel, K. Dorschel, A. Hahn and G. Muller, "Optical properties of circulating human blood in the wavelength range 400-2500 NM," Journal of Biomedical Optics 4(1), 36-46 (1999)

20. L. Wang, S. L. Jacques and L. Zheng, "MCML--Monte Carlo modeling of light transport in multi-layered tissues," Computer methods and programs in biomedicine 47(2), 131-146 (1995) 